
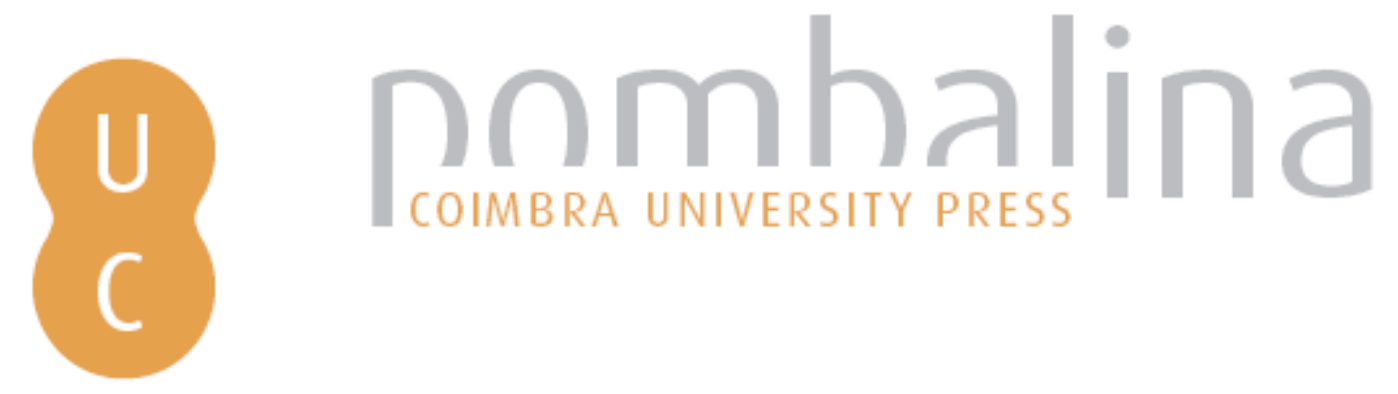

\title{
Denken, das sich (von) selbst denkt: ein automaten-theoretischer Blick auf Fichtes und Hegels Systemkonzeption
}

\author{
Autor(es): Zelger, Manuel \\ Publicado por: Imprensa da Universidade de Coimbra \\ URL \\ persistente: URI:http://hdl.handle.net/10316.2/31658 \\ DOI: $\quad$ DOI:http://dx.doi.org/10.14195/978-989-26-0205-9_23 \\ Accessed : $\quad$ 26-Apr-2023 13:28:44
}

A navegação consulta e descarregamento dos títulos inseridos nas Bibliotecas Digitais UC Digitalis, UC Pombalina e UC Impactum, pressupõem a aceitação plena e sem reservas dos Termos e Condições de Uso destas Bibliotecas Digitais, disponíveis em https://digitalis.uc.pt/pt-pt/termos.

Conforme exposto nos referidos Termos e Condições de Uso, o descarregamento de títulos de acesso restrito requer uma licença válida de autorização devendo o utilizador aceder ao(s) documento(s) a partir de um endereço de IP da instituição detentora da supramencionada licença.

Ao utilizador é apenas permitido o descarregamento para uso pessoal, pelo que o emprego do(s) título(s) descarregado(s) para outro fim, designadamente comercial, carece de autorização do respetivo autor ou editor da obra.

Na medida em que todas as obras da UC Digitalis se encontram protegidas pelo Código do Direito de Autor e Direitos Conexos e demais legislação aplicável, toda a cópia, parcial ou total, deste documento, nos casos em que é legalmente admitida, deverá conter ou fazer-se acompanhar por este aviso.

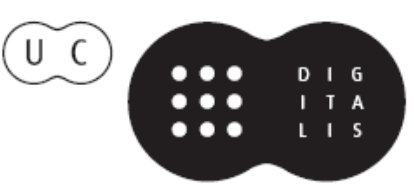


Edmundo Balsemão Pires

Burkhard Nonnenmacher

Stefan Büttner-von Stülpnagel

Editors

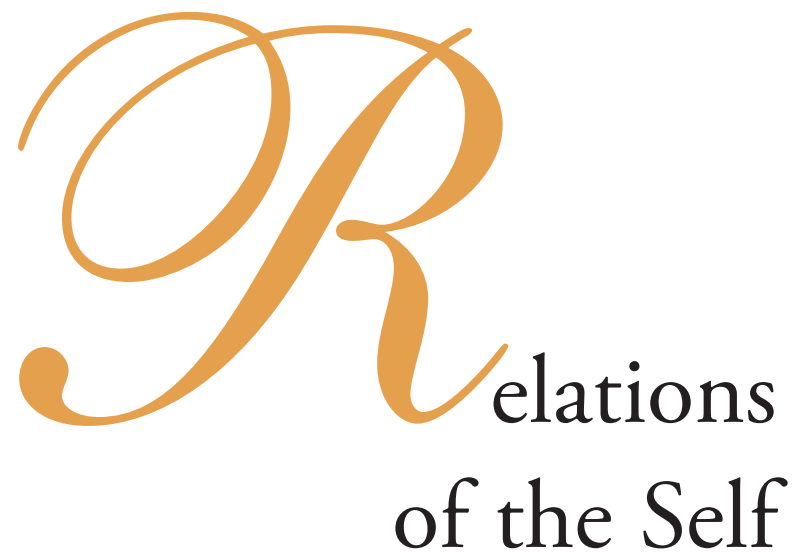




\section{DENKEN, DAS SICH (VON) SELBST DENKT EIN AUTOMATENTHEORETISCHER BLICK AUF FICHTES UND HEGELS SYSTEMKONZEPTION}

Das Thema des Denkens, das nur sich selbst denkt, also dessen Tun darin besteht, dieses sein Tun sich zum gedachten Inhalt zu machen, möchte ich im Folgenden aus der verfremdenden - aber, wie ich hoffe - fruchtbaren Perspektive angehen, die der eines Ingenieurs gleicht. Angeregt durch die Behauptungen Fichtes und Hegels, dass die Wissenschaftslehre (W.L.) bzw. die Wissenschaft der Logik (W. d. L.) nicht von Leistungen der denkenden individuellen Subjekte abhingen, sondern sich von selbst machten, geht mein besonderes Interesse dahin, zu sehen, wie das sich denkende Denken zu konstruieren sei, so dass es in seinem Vollzug sich selbst zu seinem gedachten Inhalt macht und darin sich selbst generiert. ${ }^{1}$ Den Anmarschweg über die Automatentheorie wähle ich deshalb, weil sie die Möglichkeit bietet, frei von selbstbewusstseins- und subjektivitätstheoretischen Vorannahmen diejenigen gedanklichen Operationen in den Blick zu bekommen, die es vermögen, ein sich selbst generierendes System zu konstruieren, oder besser gesagt sich konstruieren zu lassen. Als zu untersuchende Systeme habe ich nur die W. d. L und die W.L., hier vor allem die Darstellungen von 1811 und 1812 im Blick. Als Prozessoren dieser Tätigkeit gelten dann nicht mehr die die W.L. oder die W. d. L. betreibenden Subjekte. Ihnen kommt nach Auskunft Fichtes und Hegels ein lediglich passiver zuschauender Status zu. ${ }^{2}$

Was dieses autonome Operieren der W.L. und der W. d. L. mit Automaten vergleichbar macht, ist zunächst einmal die abstrakte Eigenschaft, dass sie, nachdem sie ihren Betrieb aufgenommen haben, eine endliche Menge von Schritten in einer notwendigen Reihenfolge bis zum definiten Endschritt durchlaufen. Es sei hier bereits darauf aufmerksam gemacht, dass die Formulierung vom Durchlaufen verschiedener Schritte nur eine unzureichende

1 "Automat" wird hier im ursprünglichen Sinne von "sich selbst bewegen" verstanden. Vgl. Oswald Wiener, Manuel Bonik, Robert Hödicke, Eine elementare Einführung in die Theorie der Turing-Maschinen, Wien - New York, 1998, S. 1.

${ }^{2}$ Vgl. Johann Gottlieb Fichte, Wissenschaftslehre aus dem Jahre 1812, in: Lauth, Reinhard, u. a., J.-G.-FichteGesamtausgabe der Bayerischen Akademie der Wissenschaften, Bd. II/13, Stuttgart 2002, S. 46: „Die Einsicht macht sich selbst, und nur insofern ist sie richtig. Was sich nicht selbst macht, was irgend ein Ich hindenkt, ist falsch."; Georg Friedrich Wilhelm Hegel, Enzyklopädie der philosophischen Wissenschaften im Grundrisse, in: Werke, Bd. 8, Frankfurt a. M. 1986, $\$ 41$ Zusatz 1: "Die Denkformen müssen an und für sich betrachtet werden, sie sind der Gegenstand und die Tätigkeit des Gegenstandes selbst; sie selbst untersuchen sich...". 
Beschreibung der Betriebsart besagter philosophischer Systeme ist. Für die Durchführung meiner Absicht, die W.L. und die W. d. L. als Automaten zu betrachten, kann diese Ungenauigkeit vorerst hingenommen werden. In einem 1. Schritt möchte ich, so knapp als möglich, die Komponenten und Funktionsweisen endlicher Automaten präsentieren, um dann in den folgenden drei Schritten, die dann auf die herausgestellten wesentlichen Komponenten und Funktionen zurückgreifen, einen Vorschlag zu unterbreiten, wie Automaten konstruiert sein müssen, deren Betrieb sich als die Entwicklung der Systeme der W.L. und der W. d. L. verstehen lassen. Ein bereits hier vorwegzunehmendes, nicht zu unterschätzendes Ergebnis der Auseinandersetzung mit den Grundprinzipien der Automaten ist, dass sich das Prozessieren des sich selbst denkenden Denkens nicht mit Algorithmen rechnender Systeme in Einklang bringen und folglich sich auch nicht in den Rahmen einer mathematisierten Logik einspannen lässt.

\section{Endliche Automaten ${ }^{3}$}

Die leitende Konstruktionsidee für endliche Automaten besteht darin, dass sie während ihres Betriebes in eine endliche Anzahl von diskreten und immer in eindeutiger Weise aufeinander abfolgenden Zuständen versetzt werden. Während der Anfangszustand in der benannten endlichen Zustandsreihe bereits impliziert ist, bedarf die Festlegung des Endzustands weiterer Bestimmungen ${ }^{4}$. Festgelegt wird der Endzustand mittels einer weiteren Komponente endlicher Automaten. Der Zustandswechsel geschieht nämlich so, dass die Automaten erst in Abhängigkeit eines eingegebenen Datums in den Nachfolgezustand eintreten. Beschrieben werden kann ein solcher Zustandsübergang mittels einer zweistelligen Funktion, deren Argumente die Bezeichnung eines Zustandes und der Typ eines Eingabetokens sind, und die auf den Folgezustand als dem Wert der Funktion abgebildet werden. Der Endzustand ist dann dadurch ausgezeichnet, dass er keine Eingaben mehr entgegennimmt. ${ }^{5}$

${ }^{3}$ Der Referenztext für die folgende Darstellung ist: John E. Hopcroft, Rajeev Motwani, Jeffrey D. Ullman, Einführung in die Automatentheorie, Formale Sprachen und Komplexitätstheorie, 2. Aufl. München 2002.

${ }^{4}$ Die Theorie endlicher Automaten kennt mehrere akzeptierte Zustände. Für die automatentheoretische Sichtweise auf die Systeme der W.L. und der W. d. L. ist ein definitiver Endzustand von entscheidender Bedeutung. Die Konstitutivität eines einzigen Endzustands wird deshalb von den Turingmaschinen übernommen.

${ }^{5}$ Um die Weise, wie die Übergangsfunktion den Zustandswechsel regelt, deutlich zu machen, sei ein kleiner Beispielautomat konstruiert. Er übernimmt eine für endliche Automaten übliche Aufgabe. Er soll keine weitere Eingabe mehr entgegennehmen, wenn eine Ziffer "0" gefolgt von einer Ziffer "1" eingegeben wurde. Durch die Übergangsfunktion: $\Phi($ Zustandsbezeichnung, Eingabetoken $) \rightarrow$ Zustandsbezeichnung, die durch die folgende Tabelle:

\begin{tabular}{c|c} 
Argument & Wert \\
\hline$<\mathrm{Z}_{0}, 0>$ & $\mathrm{Z}_{1}$ \\
\hline$<\mathrm{Z}_{0}, 1>$ & $\mathrm{Z}_{0}$ \\
\hline$<\mathrm{Z}_{1}, 0>$ & $\mathrm{Z}_{1}$ \\
\hline$<\mathrm{Z}_{1}, 1>$ & $\mathrm{Z}_{2}$
\end{tabular}

definiert ist, wird der Automat vollständig bestimmt. $Z_{0}$ bezeichnet den Anfangs-, $Z_{2}$ den Endzustand. Da bei fortlaufender Eingabe einer Ziffer "1" die Ziffernfolge "01" nicht (ein)gegeben worden sein kann, verbleibt der Automat im Anfangszustand $\left(\Phi\left(Z_{0}, 1\right)=Z_{0}\right)$ Hingegen ist bei einer Ziffer "0" die erste Bedingung für das Eintreten 
Was Automaten der beschriebenen Art prinzipiell auszeichnet, ist ihr Prozessieren durch Zustandswechsel, der in Abhängigkeit von (Ein)Gegebenem in eindeutiger Weise erfolgt. Deswegen soll der Übergang von Zustand zu Zustand näher betrachtet werden. Damit der Automat in seinem Betrieb bei Eingabe eines Tokens in den Folgezustand findet, muss im Automaten die durch die Übergangsfunktion ausgedrückte Verknüpfung in einer vom Automaten vollziehbaren Weise niedergelegt sein. Da ein Zustand an sich selbst einzig dadurch bestimmt ist, eine Eingabe entgegennehmen zu können, muss er seine Qualifizierung als Vorgängerzustand für einen Folgezustand von außen erhalten. Dies geschieht dadurch, dass die Zustände in eine lineare räumliche Ordnung gebracht und darin jeweils als Vorgänger und Nachfolger verknüpft werden. Der Betrieb des Automaten ist dann so organisiert, dass bei Vorliegen einer Eingabe in einem bestimmten Zustand mittels einer Bewegung entlang der verknüpfenden Verbindung der Zustand aufgefunden wird, mit dem der Betrieb fortsetzbar ist. Die durch räumliches Nebeneinandergestelltsein realisierte Verknüpfung eines Zustandes nach einer Eingabe mit seinem Folgezustand kann in den Reihen der die Übergangsfunktion $\Phi$ bestimmenden Tabelle in Anm. 5 direkt abgelesen werden. ${ }^{6}$ Außer dieser Verknüpfung, einer Einordnung in die Stellen eines Vorher und eines Nachher besitzen die Zustände keine intrinsischen Bestimmtheiten, die den Übergang zum Folgzustand aus diesen Bestimmtheiten notwendig machen würden. Die Zwangsläufigkeit, mit der der Automat bei Eingabe eines Tokens von einem Zustand genau zu einem Folgzustand fortschreitet, ist nichts anderes als der durch die Verknüpfungen festgelegte Weg entlang der mit Daten versehenen Zustände. Der Zwang resultiert daraus, dass der Weg einzig linear verläuft und keine Bifurkation zulässt. Die lineare Reihung ist der Zwang.

Die Verknüpfung der Zustände aber, die dem Automaten seinen streng determinierten Ablauf garantiert, ist selbst kein Resultat des Betriebs, sondern ihm in Form der räumlichen Stellenordnung vorgegeben. Gleichgültig wie reichhaltig das Alphabet, also die Vielfalt der eingebbaren Datentypen ist, oder wie viele Zustände verknüpft werden, der in nebeneinander geordnete Stellen quantifizierte Raum bleibt die identische Voraussetzung für jeden Automaten.

Die in der Automatentheorie abgehandelten Automaten hängen also von drei Voraussetzungen ab, die ihr Betrieb nicht garantieren kann: (1) der zur Stellenordnung quantifizierte Raum (der Laufzeitumgebung), (2) der Verknüpfung eines Paares - bestehend aus einer Zustandsbezeichnung und einem Typ von Eingabetoken - mit einem Nachfolgezustand (dem Programm) und (3) den Eingabetokens (den Daten). Dass die Zustandsverknüpfungen, die das Programm regelt, von der Laufzeitumgebung des in Leerstellen quantifizierten Raumes abhängen, ist bereits aus dem Vorhergehenden bekannt. Aber auch die Daten setzen den in Stellen quantifizierten Raum voraus. Die Möglichkeit des Programms den Übergang von einem Zustand zu seinem Nachfolgerzustand aufgrund des Eingabetyps zu regeln, hat zur Voraussetzung, dass die Typen der Eingabetoken

\footnotetext{
des Endzustands erfüllt. Werden nun weiterhin Ziffern vom Typ "0" eingegeben, dann kann es nicht zum Eingegebensein der Folge "01" kommen, also der Automat auch nicht in den Endzustand eintreten $\left(\Phi\left(Z_{1}, 0\right)=Z_{1}\right)$. Erst mit der Eingabe einer Ziffer "1" im Zustand $Z_{1}$ kann der Endzustand eintreten $\left(\Phi\left(Z_{1}, 1\right)=Z_{2}\right)$.

${ }^{6} \mathrm{Ob}$ dieses räumliche Nebeneinander mittels der Positionierung von Vorgänger- und Folgzuständen in den Spalten einer Tabelle, an den Knoten eines Ablaufdiagramms oder in den Speicheradressen eines Computers realisiert wird, ist dabei gleichgültig.
} 
unterschieden werden können. Typisiert sind die Eingabetokens durch ihre Figürlichkeit, also durch die Konstruktionsvorschrift geformter Flächen. ${ }^{7}$ Die Zustände, die bis auf den Endzustand dadurch ausgezeichnet sind, dass sie Eingaben entgegennehmen, müssen demnach selbst eine räumliche Komponente besitzen. Verarbeitet werden kann eine Eingabe nur dann, wenn sie im Automaten zu stehen kommt. Zustände können daher als Leerstellen aufgefasst werden, von denen aus, wenn in ihnen ein Token des im Alphabet erlaubten Typs zu stehen kommt, zu einer aufnahmebereiten Leerstelle fortgeschritten wird, sofern eben nicht der Endzustand erreicht ist. ${ }^{8}$

Was die Automaten der Automatentheorie also wesentlich charakterisiert, ist die Eigenschaft, dass ihr Prozessieren als Bewegung, als Fortschreiten im wörtlichen Sinne, als an den Raum gebundene extendierte Zeit verstanden werden muss. Die Raumstelle, die der Zustand ist, ist in ihrer Statik, Fixiertheit die Bedingung der Möglichkeit, dass zu einer ebenfalls fixierten Stelle fortgeschritten werden kann. Zudem garantiert sie, die Unterscheidbarkeit der eingegebenen Tokens als figurierte Flächen. Mit Hilfe der Tatsache, dass die Statik der Raumstellen aktuell nur genau eine Belegung zulässt, können die Zustände in eine eindeutige Reihenposition gebracht werden. Die erste für die Aufnahme einer Eingabe bereitgestellte Leerstelle des in Anm. 5 präsentierten Beispielautomaten ist deshalb die erste, weil sie bei Aufnahme einer "1" für die Aufnahme der Folgeeingabe mit sich selbst verknüpft ist und bei Aufnahme einer "0" mit einer Leerstelle verknüpft ist, die bei Aufnahme einer "0" anders verknüpfen muss.

Der in Leerstellen quantifizierte Raum ist demnach die Grundvoraussetzung der beiden anderen Voraussetzungen, die man für endliche Automaten machen muss, damit sie sich als konstruierbar erweisen. Änderung, Entwicklung, der Wechsel der Zustände vom Anfangsbis zum Endzustand manifestiert sich als Bewegung im Rahmen der für den Ablauf vorgegebenen Umgebung. Deren Eigenschaften, die vom Automaten nicht errechnet werden, garantieren den eindeutigen Fortgang der Prozesse aufgrund festgefügter Bahnen in dem durch diese prägbaren, in Stellen quantifizierten Raum. Die Unverwechselbarkeit, Unaustauschbarkeit der Raumstellen, die die Statik des Raumes ausmachen, garantieren in ihrem Nebeneinander die Notwendigkeit des Fortschreitens im Betrieb des Automaten.

Die nun anzugehende automatentheoretisch angeleitete Betrachtung der Systeme der W.L. und der W. d. L. lässt sich von der Automatentheorie die Themen Anfang, Übergang

${ }^{7}$ In den Automaten der elektronischen Datenverarbeitung wird diese Figürlichkeit durch das Muster geladener und nicht geladener Transistoren realisiert. Auf der physikalischen Ebene unterliegen der geladen/nicht-geladenDisjunktion Grenzwerte, die nicht unterschritten bzw. überschritten werden dürfen.

${ }^{8}$ Für den mit Hilfe von Stift und Papier ausführbaren Beispielautomaten aus Anm. 5 erkennt man dieses im Automaten Stehen auf einen Blick, wenn man die Zustände als durch Indizes typisierte Leerstellen interpretiert, die bei Befüllung mit einem bestimmten Token des Alphabets wiederum mit einer indizierten Leerstelle verknüpft werden. Die die Übergangsfunktion definierende Tabelle aus Anm. 5 stellt sich dann so dar:

$[0]_{0} \rightarrow[]_{1}$

$[1]_{0} \rightarrow[]_{0}$

$[0]_{1} \rightarrow[]_{1}$

$[1]_{1} \rightarrow[]_{2}$

Der Endzustand nimmt keine Eingabe mehr an und besitzt deshalb auch keine auszufüllende Leerstelle, obwohl er als Ende der durchlaufenen Zustände räumlich ausgezeichnet ist. 
und Ende vorgeben. Auf nachweisende Zitate wird im Folgenden größtenteils verzichtet, da es auf die systematische Problemstellung ankommt, von der allerdings angenommen wird, dass sie auch die Fichtes und Hegels war.

\section{Der Anfang}

Bei der Konstruktion der von der Automatentheorie beschriebenen Automaten stellt sich der Anfang des Prozessierens nach dem bisher Gesagten als trivial dar. Bereits das diesen Automaten unterliegende Konzept des sequentiellen Abarbeitens der im Programm vorgeschriebenen Stationen erzwingt einen Anfang und ein Ende.

Die besondere Herausforderung für die Konstruktion von Automaten, die die Systementwicklung der W.L. oder der W. d. L. modellieren soll, besteht darin, dass eine Umgebung, in der Programm und Leerstellen für Daten zur Verfügung stehen, nicht vorausgesetzt werden darf. Es muss darauf verzichtet werden, die Stationen vorab in eine solche Reihenfolge zu bringen, dass die potentiellen, datenabhängigen Abläufe gesichert sind, bevor der Automat de facto anläuft. Die bisher beschriebenen Automaten sind konstitutiv darauf angewiesen, dass sie in Form des Programms und in Form des Repertoires der Typen einzugebender Daten vorliegen und im Lauf des Betriebs diese Form auch nicht mehr ändern können. Diese starre, mit sich identisch bleibende Form, in der sich der Automat während der Abarbeitung des Programms bewegt, verdankt er seinem environment, dem Raum. Der Automat wird zu einem bestimmten Automaten dadurch, dass in dem zu Stellen quantifizierten Raum die Verknüpfung der Daten-annehmenden Leerstellen in Abhängigkeit der dort zu stehen kommenden Figuren oder Muster von außen eingeschrieben werden. Den Betrieb aufnehmen und fortsetzen kann der Automat auch nur dann, wenn ihm die Daten ein- bzw. vorgegeben werden. Der Anfang wird folglich durch eine Aktion getätigt, die nicht aus dem Lauf des Automaten selbst stammt.

Die Herausforderung anzunehmen, einen Automaten zu konstruieren, der seinem Betrieb nicht vorausliegt, also der Forderung aller nicht dogmatisch verfahrenden Philosophie nachzukommen, keine für das System konstitutive Bestimmtheit anzuerkennen, die nicht im System selbst erzeugt ist, ist nur dann möglich - so meine These -, wenn man sich von der Raumbindung und der mit ihr verbundenen starren Identität radikal befreit. Man muss, um mit Spinoza zu sprechen, vom Attribut der extensio auf das der cogitatio umschalten. Identität und Raum hängen deshalb so eng zusammen, weil in eine Raumstelle nur eintreten kann, was vorher an einer anderen Stelle war. Die Daten selbst werden im Lauf des Automatenbetriebs nicht verändert, sondern nur deren Stellung im Raum. Sie bleiben so, wie sie unmittelbar gegeben sind. Die Unverwechselbarkeit des räumlich Vereinzelten beruht darauf, dass dasjenige, was an der einen Stelle ist, nicht zugleich an einer anderen Stelle präsentiert werden kann. Es kann von anderem auseinander gehalten werden. Etwas und Anderes sind voneinander abgegrenzt und der Wechsel von einem zum anderen ist durch das Kreuzen der Grenze markiert, womit ausgeschlossen wird, dass es zur Verwechslung kommt. ${ }^{\text {? }}$

${ }^{9}$ Mit den Begriffen "Kreuzen", "Grenze" und "Markieren" ist bewusst auf George Spencer Brown, Laws of Form, New York 1979, angespielt. Hier ist die Rückführbarkeit alles Rechnens auf Bewegung in besonders radikaler Form vollzogen. 
Aus dem bisher Ausgeführten lassen sich bereits Konsequenzen ziehen, wie nach dem Wechsel in die Umgebung des Denkens die dort operierenden Automaten nicht konstruiert werden dürfen, sollen sie die Anforderung des voraussetzungslosen bzw. umgebungsfreien Anfangs erfüllen. Es kann ein solcher Automat nicht so konstruiert werden, dass er als fix bestimmtes subiectum seinen Operationen unterlegt wird. Die letztgültige Basis des so Vorgegebenen sind belegte Raumstellen, auf die indexikalisch verwiesen wird. ${ }^{10}$ Dieses Vorliegende würde den Anspruch auf Voraussetzungslosigkeit und die Selbstbestimmung des Systems bereits im Ansatz konterkarieren.

Zudem darf das gedankliche Operieren nicht so begriffen werden, dass von Gedanke zu Gedanke nach einer ihnen äußerlichen Regel fortgeschritten wird. Die Gedanken liegen nicht als Vereinzelte, z. B. per Schrift räumlich extendierte Sätze, vor, um dann durch ihnen äußerliche Verknüpfungen in einen linearen Ableitungszusammenhang gebracht zu werden. Die Operationen im Bereich des reinen Denkens als der Raumbindung radikal entzogenem sind - und das mag überraschen - kein Fortschreiten von Prämissen zu Konklusionen nach ihnen äußerlichen Regeln der Schlusslehre. Nicht nur die über Hinzufügen, Austauschen und Wegnehmen von Zeichenketten fungierenden Kalküle des natürlichen Schließens manifestieren sich als Bewegung von an Raumstellen Vorgegebenem, sondern auch in dem auf Aristoteles zurückgehenden Syllogismus kommt durch die Stellung der Terme in den Prämissen und die Fixierung von Subjekt und Prädikat in Raumstellen die Notwendigkeit des Schließens zustande. ${ }^{11}$ Die Schlüsse operieren also auf Vorgegebenem, das in seiner räumlichen Position zwar geändert werden kann, aber gerade deshalb seine fixe Identität erhält. Die den größeren, mittleren und kleineren Term determinierenden Begriffe bleiben in der Schlussfigur dieselben. Auch die in die Schlüsse als Prämissen eingehenden Urteile erscheinen in der formallogischen Darstellung: "S ist $\mathrm{P}$ " als durch Raumstellen identifizierbare. In der Verkettung des vorgegebenen Subjektausdrucks mit dem vorgegebenen Prädikatausdruck mittels der Kopula wird die Prädikation als Operationsmodus identifizierbar.

Ein Bestimmtes liegt dann als logisches Subjekt bereits vor und das Prädikat, das hierfür auch schon fertig vorliegt, wird dann als eine weitere Bestimmtheit an es herangetragen. Da die formale Logik ihre Beschreibung der cogitatio an die extensio bindet, ist sie ungeeignet, der Konstruktion des voraussetzungslos operierenden Automaten Hilfestellung zu leisten.

Mit dem Verzicht auf Vorgegebenes stellt sich der Konstruktion des voraussetzungslos und umgebungsfrei operierenden Automaten ein zusätzliches Problem. Da die Operationalität im raumgebundenen Vorgegebenen sich immer als Bewegung ausprägt, ermöglicht sie es,

${ }^{10}$ Für die Festlegung des in Anm. 5 beschriebenen Beispielautomaten werden die Zustände und Daten als Mengen angegeben, indem auf deren Elemente gezeigt wird, die innerhalb der geschweiften Klammern zu stehen kommen:

Alphabet $A:=\{0,1\}$

Zustände $\mathrm{Z}:=\left\{\mathrm{Z}_{0}, \mathrm{Z}_{1}, \mathrm{Z}_{2}\right\}$.

Das Zeichen ":=" muss demnach so verstanden werden, dass es besagt: Dort stehen die Typen des Alphabets bzw. die Bezeichnungen der Zustände.

${ }^{11}$ Fichte und Hegel haben sich explizit dagegen ausgesprochen, dass die formale Logik einen adäquaten Zugang zu den Operationen der cogitatio anzubieten vermag. Vgl. Johann Gottlieb Fichte, Über das Verhältnis der Logik zur Philosophie oder Transcendentale Logik, Hamburg 1982, passim; Georg Friedrich Wilhelm Hegel, Wissenschaft der Logik, Hauptwerke in sechs Bänden, Bd. 3, Hamburg 1999, S. 36. 
selbst wieder als räumlich dargestellt und dadurch als Bestimmtes identifiziert werden zu können. An der darstellenden bzw. regelnden Funktion des Programms für das Prozessieren des in Anm. 5 dargestellten Beispielautomaten kann der Bezug des Nebeneinanders im Programm auf das Nacheinander im Programmablauf exemplifiziert werden. Der Betrieb des Automaten bei der anfänglichen Eingabe einer "0", zeitlich gefolgt von der einer weiteren " 0 " und der einer " 1 " kann nach der in Anm. 8 verwendeten Programmnotation dann wie folgt dargestellt werden:

$[0]_{0} \rightarrow[0]_{1} \rightarrow[1]_{1} \rightarrow[]_{2}$

Die Gerichtetheit des Pfeils, der zwischen den Bezeichnungen der Zustände steht, bewirkt, dass das Nacheinander des Operierens durch das Nebeneinander der Raumstellen identifiziert und wie jetzt geschehend zum Gegenstand von Prädikaktionen gemacht werden kann. Erkennen kann man hieraus auch, wie die tabellarische Programmnotation den Ablauf des Programms regelt. Die Beschreibung des Ablaufs zeigt, dass die einzelnen Schritte nichts anderes sind als der Vollzug einzelner Tabellenzeilen.

In der Satzform "S ist P" liegt ein vergleichbarer Fall vor, wie durch Nebeneinanderstellung ein Prozess - hier das Bestimmtwerden des Subjekts durch das Prädikat identifizierbar ist.

Insofern stellt sich der Konstruktion des voraussetzungslos operierenden Automaten die Aufgabe, dass dessen Operieren zu beschreiben bzw. zu regeln ist, ohne sich der Umgebung des in Stellen quantifizierbaren Raumes bedienen zu können. In der cogitatio muss Tätigkeit zu erfassen und regeln sein, die nicht Bewegung ist. Die Erfassung bzw. Regelung kann dem zu konstruierenden Automaten nicht wie bei den beschriebenen Zustandsautomaten in Form des als Nacheinander fassbaren Nebeneinanders vorgegeben werden. Die Auszeichnung eines Anfangs z. B. mit Hilfe des Urteils "x ist der Anfang von y" bezöge sich auf ein bestimmtes Vorgegebenes, das mittels des Nebeneinanders im Urteilsausdruck als Nacheinander verstanden werden kann: Dem Anfang folgt das, für das er Anfang ist.

Für die Konstruktion des voraussetzungslos operierenden Automaten der cogitatio heißt das, dass nichts als sein Anfang zu konstatieren ist, da dies nur mittels Prädikation geschehen könnte, aber 1.) Urteile im Subjektausdruck auf Vorgegebenes rekurrieren, und 2.) sie selbst nicht das sind, worüber sie urteilen, also im environment des intendierten Automaten zu platzieren wären. In dem Moment, in dem etwas als Anfang bestimmt ist, stellt sich das Urteil außerhalb des durch es beurteilten Sachverhalts.

Die einzige Konsequenz, die aus dem zu den Problemen der Konstruktion eines voraussetzungslosen Beginnens Ausgeführten gezogen werden kann, ist, mit nichts Bestimmtem $\mathrm{zu}$ beginnen und somit überhaupt auf einen als solchen zu bestimmenden Anfang zu verzichten. Was wie Resignation aussieht, ist die Inbetriebnahme der von der extensio befreiten cogitatio. Wird auf Bestimmtes verzichtet, so steht nichts zur Verfügung mit dem etwas - im wahrsten Sinne des Wortes - anzufangen wäre. Würde diese absolute Unbestimmtheit aber als Anfang bestimmt werden, so wäre sie zum Bestimmten gemacht worden, das dieser seiner Bestimmung vorgegeben ist. Die Aufgabe, einen Automaten zu konstruieren, der umgebungsfrei operiert, kann also dahingehend spezifiziert werden, dass anzugeben sei, wie das Unbestimmte als nicht Bestimmtes gefasst werden kann. Die Frage nach dem Wie ist in automatentheoretischer Perspektive die Frage nach dem Verfahren, nach dem Programm. 
Will man das anzugebende Verfahren als Prädikation modellieren, so stellt sich die Bauweise des Begriffs "unbestimmt" in den Weg. Seine Bedeutung ist, nicht als Prädikat verwendet werden zu können, da es die Funktion des Prädikats im Urteil ist, auf etwas bezogen zu sein, das der Bestimmung durch das Prädikat vorgegeben ist. ${ }^{12}$ Das Unbestimmte als nicht bestimmt, also adäquat, zu erfassen, ist demnach einzig möglich, wenn gar nicht geurteilt wird, also nicht erfasst wird. Nicht zu bestimmen, ist nicht dasselbe wie prädizieren, dass etwas nicht bestimmt ist. Auch das negative Prädizieren das Absprechen eines Prädikats unterstellt Vorgegebenes, das dem Prädikat nicht subsumiert, dem das Prädikat abgesprochen wird.

Deshalb darf das Erfassen durch Nicht-Erfassen nicht so ausgeführt werden, nicht so gedacht werden, dass man die Aussage, das Unbestimmte sei nicht erfassbar, für dieses Erfassen ausgibt. Wie unschwer zu erkennen ist, dominiert hier erneut die Urteilsform, obwohl sie doch außer Geltung gesetzt werden soll. Das Unbestimmte steht nicht als Datum einem Programm zur Verfügung, das jedes Prädikat in Bezug auf dieses Datum als unzutreffend qualifiziert.

Das Unbestimmte ist nichts anderes als das Programm, das in Ermangelung eines Ausgangszustands (eines der Prädikation zur Bestimmung Vorgegebenen) und eines Folgezustands (des Bestimmtseins durch das Prädikat) in der Leere des Nicht-Prädizierens, Nicht-Bestimmens besteht. In der Aussage, dass das Unbestimmte das Programm sei, das nicht bestimmt, findet aber wiederum eine Prädikation statt.

Dem darin getätigten Rekurs auf ein Vorgegebenes kommt man nur dann aus, wenn die Aussage nichts aussagt und somit selbst nur nichtssagender Leerlauf ist. Das Nicht-Bestimmen kann als umgebungsfrei, sprich absolut, operierendes keiner Prädikation unterworfen werden. Nicht-Bestimmen sagt eben nichts aus. Unbestimmtes im Sinne von Nicht-Bestimmen kann somit nicht identifizierbares Eines sein, das durch die Bestimmtheit "Nicht-Bestimmen" bestimmbar wäre. "Nicht-Bestimmen" ist Nicht-Bestimmtheit, bestimmt nichts und ist somit nur die Leere des Nicht-Bestimmens selbst. Das absolute Nicht von allem Bestimmen, Identifizierbaren ist selbst nicht zu identifizieren und vernichtet seine Identität als Gegenüber zu allem Bestimmten im Moment seines Operativwerdens. In der Vernichtung seiner Identifizierbarkeit macht sich das Nicht-Bestimmen erst, da es hiermit sich dem auf Vorgegebenes gerichteten identifizierenden Zugriff entzieht und somit selbständig seine Unbestimmtheit ausführt. Das Nicht-Bestimmen ist operativ, indem es das "ist" annihiliert, zum Nicht-Bestimmen macht. Das Programm ist seiner Ausführung nicht als geschriebenes vorgegeben, sondern macht sich in seiner Ausführung selbst, indem es einzig ausführt, diese seine (Be)Schreibung als nichtsagend auszuzeichnen.

Wenn die Befreiung von der Bindung an den Identifizierung erlaubenden Raum zugleich auch Befreiung von der Urteilsform bedeutet, dann stellt sich die Frage, wieso das NichtBestimmen überhaupt als cogitatio, als Denken firmieren kann. Die Frage soll beantwortet

\footnotetext{
${ }^{12}$ Auch hierin vollzieht sich die Befreiung von der Raumbindung, denn die Beziehbarkeit des Prädikats auf Vorgegebenes wird in einer langen Tradition darin gesehen, dass der Bereich des Vorgegebenen aufgeteilt wird in diejenigen, die unter den vom Prädikat ausgedrückten Begriff fallen, und diejenigen, die nicht darunter fallen. Dass Begriffsverhältnisse somit durch Konstellationen von begrenzten Flächen (z. B.: Venn-Diagrammen) darstellbar sind, nimmt nicht Wunder. Um nicht nur auf den augenscheinlichen Fall der Verbindung der Prädikatenlogik mit Mengenlehre zu verweisen, sei als ein weiterer Beleg auf die Jäsche-Logik verwiesen: Immanuel Kant, Logik, Immanuel Kant Werkausgabe, hrsg. von Wilhelm Weischedel, Bd. 6, Frankfurt a. M. 1977, S. 534 und S. 539.
} 
werden, indem gezeigt wird, dass Denken, das sich selbst denkt, um das Denken von Unbestimmtem gar nicht herumkommt und somit in den zuvor ausgeführten Prozess gezogen wird.

Wenn man einer langen Tradition folgend davon ausgeht, dass Denken, die cogitatio, in Betrieb Prädizieren ist, dann stellt sich die Frage, ob die Prädikate, die dem Prädizieren selbst als Gegenstand, als logisches Subjekt Genommenes zugesprochen werden können, einzig durch die mittels Raumgebundenheit identifizierbare Satzform "S ist P." vermittelt sind. Dann wäre Denken des Denkens nichts anderes als Denken geradehin, fremdreferentielles Denken. Dass die Prädikation als Gegenstand der Prädikation nicht in der Konkatenation eines Subjektausdrucks mit einem Prädikatausdruck, ihrer Satzform aufgeht, sieht man schon daran, dass Urteile auch über das in Urteilen Gemeinte, ihre semantische Dimension gefällt werden können. Die Eigenschaft des Urteils, sich auf einen Sachverhalt zu beziehen, ist keine, die unmittelbar den Ausdrücken zukommt, die das Urteil ausdrücken. Was die Bestimmtheit ist, die zugesprochen wird, und was das ist, dem sie zugesprochen wird, kann aus dem Ausdrucksgefüge entnommen werden. Was hingegen das Sein im So-Bestimmtsein des zu Bestimmenden ausmacht, kann nicht im Gefüge von Subjekt und Prädikat untergebracht werden. Wollte man formulieren, in dem Urteil "Die Rose ist rot." sei gemeint, dass die Rose von der Bestimmtheit "rot" aber auch von der Bestimmtheit "Sein" sei (!), so nimmt man erneut Sein in Anspruch, das nicht in der Bestimmtheit "Sein" gemeint sein kann. Das Bestimmtsein oder Nicht-Bestimmtsein durch das Prädikat "Sein" ist nicht mittels des Prädikats "Sein" ausgesagbar. Zu sagen, die Rose sei Seiendes, sagt nicht mehr aus als: "die Rose ist ...". Darin verbleibt aber das, was das "ist" ist, was es zur Bestimmtheit der Rose beiträgt, unbestimmt. Das in Urteilen gemeinte Sein des durch Subjekt und Prädikat ausgedrückten Sachverhalts kann also mittels Prädikation keine Bestimmtheit verliehen werden. Während also auf die copulative Funktion der Kopula in der extensio der Satzform ohne weiteres mittels Prädikation Bezug genommen werden kann, entzieht sich die ontologische Funktion diesem Zugriff. Ein Satz der Form "Ist ist X.", wobei für "X" jedes Prädikat eintreten kann, offenbart diesen Entzug in augenscheinlicher Weise, wenn "Ist" in der ontologischen Funktion verstanden wird. ${ }^{13}$ Ein wesentliches Konstituens des Urteils kann also in dem bloß als Verhältnis von Subjekt und Prädikat konzipierten und über die Satzform identifizierbaren Urteil nicht aufgefasst werden. Wenn Fassen aber selbst nur die Feststellung ist, dass das mittels des Subjekts Identifizierte durch

${ }^{13}$ Zur Unterscheidung von "copulativer" und "ontologischer" Funktion vgl.: Bruno Baron von FreytagLöringhoff, Logik, Ihr System und ihr Verhältnis zur Logistik, Stuttgart 1966, S. 60. An den aus der Auseinandersetzung um den ontologischen Gottesbeweis bekannten Slogan: "Sein ist kein Prädikat." darf hier zurecht erinnert werden. Ein Blick auf die Debatten um den Begriff der Wahrheit belehrt darüber, dass die prädikative Unbestimmbarkeit des Seins nicht nur ein Problem aus dem Gebiet der Metaphysik ist. Gleichgültig welcher Theorie der Wahrheit man anhängt, jeder stellt sich das Problem, wie das gefasst werden kann, worauf sich ein Urteil bezieht und das so sein kann, wie das Urteil sagt oder nicht. Das "So" ist nur fassbar in Abhängigkeit von Subjekt und Prädikat des Urteils. Das "Sein" bereitet die benannten Schwierigkeiten. In der von den meisten Wahrheitstheorien anerkannten Konventition: "'p' ist wahr gdw p" wird auf der rechten Seite des Bikonditionals die ontologische Funktion der Kopula in Anspruch genommen, ohne dass die Konvention deren Bestimmtheit in prädikativer Form auszudrücken vermag. Die Bestimmtheit, dass der in p gemeinte Sachverhalt eine Tatsache ist, ist in der ontologischen Funktion der Kopula schon mitgemeint und bleibt als zusätzliche Bestimmtheit insofern leer. 
die vom Prädikat ausgedrückte Bestimmtheit bestimmt ist, kann das Sein nur als nicht fassbar bzw. nicht bestimmbar konzipiert werden.

Denken, das sich selbst denkt, indem es seine Vollzugsform, das Prädizieren generell thematisiert, entdeckt darin, dass es sich selbst nicht so vorgegeben ist, wie das letztendlich deiktisch Aufweisbare und über Raumstellen Identifizierbare. Wie gezeigt, führt diese Entdeckung zu der Aufgabe, das Unbestimmte als nichts Bestimmtes zu konstruieren. Hierfür muss das Denken in einen Operationsmodus umschalten, der sich nicht als Verkettung von jeweils in sich abgeschlossenen und damit identifizierbaren Prädikationen ausweisen lässt.

Bevor der von der Theorie endlicher Automaten unserer Untersuchung vorgegebene Aspekt des Übergangs betrachtet wird, seien noch einige Bemerkungen über den Bezug des Vorgetragenen zur W.L. und zur W. d. L. eingeschoben. Die Sich-Konstruktion des Unbestimmten im Leerlauf des Nicht-Bestimmens, der dieses nur ausführt, wenn er die darin zugleich auftretende Bestimmtheit "Nicht-Bestimmen" eodem actu entleert, also leerlaufen lässt, lehnt sich eng an den Anfang der W. d. L. an. Etwas anders verhält es sich mit der W.L. Die Ausgangslage scheint mir dieselbe zu sein: Das Sein ${ }^{14}$, das zumindest in den Wissenschaftslehren von 1811 und 1812 den Ausgangspunkt des systematischen Gedankengangs bildet, wird als reines In-Sich, Von-Sich, Aus-Sich beschrieben, zu dem es kein Außerhalb, kein environment gibt, in dem diese Beschreibung platziert werden könnte. Mit dieser ihm inhärerienden Umgebungsfreiheit ist der Begriff des In-Sichs ein geeigneter Kandidat, voraussetzungslos zu operieren, sofern er sich kohärent ausführen, denken lässt. Gleich der Ausgangslage der W. d. L. stellt sich das Problem von woher dem Sein die Bestimmtheit zugesprochen wird, ein reines In-Sich zu sein, zu dem es kein Außerhalb gibt. Wie bereits oben ${ }^{15}$ ausgeführt ist das Urteil nicht der in ihm intendierte Sachverhalt. Insofern muss dem Urteil ein Sein außerhalb des Seins zugesprochen werden. Damit ist aber das In-Sich, indem es ausgesprochen wird, gerade dementiert. "Indem gesagt wird; es sey nichts ausser ihm, ist etwas, eben dieses Sagen, ausser ihm." ${ }^{16}$

Die W.L. konzentriert ihre Darstellung des Ausgangsproblems der Systemgenese zwar nicht auf das der Prädikation inhärierende Verhältnis einer Bestimmtheit zum vorgegebenen Bestimmten, aber man kann das Ausgangsproblem problemlos auf die Prädikation übertragen. Das Sagen ist eine Aussage über das Sein, die das Sein so bestimmt, dass ihm keine Bestimmtheit zugesprochen werden kann, da diese immer den Ort der Intention verlangt, der nicht der des intendierten Sachverhalts ist. Es stellt sich also das Problem, wie das allem Bestimmen Entzogene als ein solches zu bestimmen sei.

So weit die gemeinsame Ausgangslage. Während aber die W. d. L. einzig das in der Bestimmtheit, unbestimmt zu sein, angelegte Programm ausführt, das in nichts anderem besteht, als dem Leerlauf des Nicht-Bestimmens, das seine Bestimmtheit, nämlich Nicht-

${ }^{14}$ Es fällt auf, dass sowohl die W.L. als auch die W. d. L. die Ausgangskonstellation ihrer Systementwicklung als "Sein" bezeichnen. Der Ausdruck steht zunächst einmal bei beiden für das dem Bereich des durch Bestimmtheiten Bestimmten und Vermittelten, if Entzogene.

${ }^{15}$ Vgl. oben S. 371.

${ }^{16}$ Johann Gottlieb Fichte, Wissenschaftslehre 1812, S. 52. 
Bestimmen zu sein, selbst noch als nicht bestimmend ausweist und sie somit als nichts bestimmen könnende Bestimmtheit leerlaufen lässt, verteilt die W.L. das Sein und das environment seiner urteilsförmigen Bestimmtheit auf zwei absolut getrennte Sphären. Diese sind einzig so aufeinander bezogen, dass von der einen nicht in die andere zu kommen ist und die eine durch die andere keine Modifikation erfahren kann. Weder kann in der Erscheinung, der Sphäre, der auch das begriffliche Bestimmen angehört, etwas sein, noch kann im Sein etwas erscheinen. Erscheinung ist nicht, Erscheinung erscheint nur. Da das Abgetrenntsein, das In-Sich-Sein immer eine Bestimmtheit ist, die eine im Urteil ausgesagte ist, bleibt die Sphäre des Urteilsunabhängigen für die W.L. zunächst einmal hypothetisch. Dass das Sein als in Prädikationen bestimmtes erscheint, nimmt die W.L. als Faktum, als Vorgegebenes hin.

Ähnlich wie die W. d. L. fragt die W.L. danach, wie die Sphäre des begrifflichen Bestimmens beschaffen sein muss, damit im Urteilen nicht immer schon das verfehlt wird, was außerhalb jedes Urteilens liegt. Im Gegensatz zur W. d. L. bleibt die Ausgangsbestimmtheit aber, das In-Sich - wenn auch hypothetisch - bestehen. Dass das, als von jedem Meinen unabhängig seiend, Gemeinte von Gnaden des Meinens sein kann, verdankt sich dem Ausgang, der das Sein als das allem Meinen Entzogene identifiziert und es so der Urteilsstruktur unterwirft. Die im Urteil getätigte Identifikation meint zwar Unabhängikeit, kann für sie aber nicht einstehen. Diesem Ungenügen des Urteils, in dem sich das Denken in der intentio recta natürlicher Weise immer schon vorfindet und aus dem es sich in der cogitatio als reinem Denken durch eine "künstliche" Konstruktion erst befreien muss, begegnet die W.L. nicht durch Verzicht auf das Urteilen, sondern indem sie aufzuzeigen unternimmt, dass die mit seinem Begriff gegebene Hypothetizität des In-Sich selbst das In-Sich zur Voraussetzung hat. Dieses Urteil, so soll demonstriert werden, ist nur möglich, weil das In-Sich seine eine Erscheinung außer sich hat. Das, was dem In-Sich als äußere Umgebung vorgegeben zu sein schien, zeigt sich als vom In-Sich Abhängiges. Wenn die W.L. auch die Bestimmtheit ihres Ausgangs, aller Bestimmtheit entzogen zu sein, nicht diesem Gesetz selbst unterstellt, indem sie am identifizierenden Urteilsvollzug festhält, so kann sie nicht dem Ausgangszustand der im environment des Raums operierenden endlichen Automaten gleichgesetzt werden. Allein schon aufgrund der Hypothetizität darf das Sein nicht als Ausgang des im Fortgang der W.L. Folgenden angesehen werden. Wie bei Behandlung des Übergangs zu zeigen sein wird, verfährt die W.L. nicht als lineare Ableitung aus am Anfang stehenden Grundsätzen. Es ist ja durchaus offen, ob das Urteil, dass das Sein der Sphäre des Urteilens entzogen sei, zur Bedingung seiner Möglichkeit hat, dass Erscheinung einzig Erscheinung des Seins ist.

Nachdem die Behandlung des Anfangsproblems für die nur im Bereich der cogitatio operierenden Automaten bereits die Auflösung des Anfangs als einer Null bzw. Eins in einer der Zahlenreihe nachgebildeten Sequenzreihe verdeutlicht hat, lässt sich if erahnen, dass auch das Konzept des Übergangs nicht das bleiben kann, was es bei den in der extensio operierenden Automaten ist. 


\section{Der Übergang}

Die Systeme der W.L. und der W. d. L. teilen mit den von der Automatentheorie beschriebenen Automaten, dass nach dem Beginn die Fortsetzung auf notwendige Weise erfolgt. Doch kann die Notwendigkeit des Übergangs nicht durch den Zwang modelliert werden, von einer bestimmten Entität aus eine Verknüpfung zu einer eindeutig bestimmten Folge-Entität herzustellen, wie das in den automatentheoretisch gefassten Automaten der Fall war. D. h., dass auch das Anschlussproblem für die umgebungsfrei und damit voraussetzungslos operierenden Automaten des reinen Denkens nicht durch das auf den Raum als seine Umgebung angewiesene Bewegungsmodell gelöst werden kann. In ihrem Vorausgesetztsein werden dort die Entitäten nämlich in ihrer Identität durch die verknüpfende Operation überhaupt nicht berührt. Es ändert nichts an einer bestimmten Leerstelle, von wo aus und wie oft sie im Betrieb des Automaten angesteuert wird. Sie ist durch die Raumstruktur ein für alle Mal identifiziert.

Vergegenwärtigt man sich die Ausführung des Programms des Nicht-Bestimmens, das einzig darin besteht, die Bestimmtheit "Nicht-Bestimmen" als leer und nichts sagend zu behandeln, und sie so gerade zu realisieren, dann lässt sich bereits erahnen, dass in dem so gearteten Prozessieren nichts Fixes gefunden werden kann, von dem aus ein als different identifizierbarer Folgezustand erreichbar wäre.

Es wurde bereits darauf hingewiesen, dass sich der Leerlauf des Nicht-Bestimmens nur um den Preis als Anfang der cogitatio auszeichnen, also als ein Nullpunkt festlegen lässt, dass der Automat still gestellt und damit die cogitatio abgebrochen wird. Auch die Sequenzierung, erst das Nicht-Bestimmen als adäquaten Operationsmodus des Unbestimmten zu konstruieren und dann diese Konstruktion des Als wiederum als inadäquat abzuweisen, bedient sich auseinanderzuhaltender Bestimmtheiten.

Nun ist zuzugestehen, dass im Vollzug der cogitatio, damit über sie überhaupt Aussagen getroffen werden können, wie es geschieht, wenn die Bestimmtheit "Nicht-Bestimmen" als nichts sagend behandelt wird, die Bestimmtheiten "nichts sagend" oder "nichts bedeutend" Bedeutung erlangen. Um dieser im Leerlauf immer wieder entstehenden Bedeutung willen, der gemäß keine Bedeutung auftreten darf, muss der Vollzug aber zugleich die Nichtigkeit, das Verschwinden der Bedeutung in Bedeutungslosigkeit, des Gesagten im Nichtssagenden sein. Unvermeidlich entsteht im Namhaftmachen dieses Verschwindens die Bestimmtheit des Nichtsagenden, Bedeutungslosen, Nicht-Bestimmenden erneut, um in Exekution ihres Programms zu verschwinden.

Dass das Entstehen und Verschwinden von Bestimmtheit im Leerlauf nicht als sukzessiv eintretende Zustände der von Raumbindung befreiten cogitatio konstruiert werden können, dafür sorgt die nicht außer Kraft gesetzte Programmatik des Nicht-Bestimmens selbst. Das Nicht-Bestimmen exekutiert sich nur in diesem Entstehen und Verschwinden. Auch das identifizierende Auseinanderhalten der Bestimmtheiten "Entstehen" und "Verschwinden" findet im laufenden Nicht-Bestimmen keinen Halt, da in der Ausführung des NichtBestimmens das Entstehen zugleich das Verschwinden ist wie das Verschwinden das Entstehen. Die im Vollzug des Nicht-Bestimmens als nicht(s) Bestimmen wiederum entstandenen Bestimmtheiten müssen nach ihrer eigenen Programmatik nun selbst wieder verschwinden. 
In einer vergleichenden, äußerlich identifizierenden Betrachtung kann man formulieren, dass die Begriffe in der cogitatio einem solchen Bauplan folgen, dass sie als Programme sich selbst als Daten realisieren. Erst indem die im Vollzug des Nicht-Bestimmens als nicht(s) Bestimmen entstandenen Bestimmtheiten "Entstehen" und "Verschwinden" selbst verschwinden, haben sie sich als nicht Vorgegebene realisiert.

Die in der nur auf sich operierenden cogitatio generierte Zeitlichkeit ist keine linear fortschreitende, über durchlaufene, auseinanderzuhaltende Zustände zählbare, sondern das Kontinuum des nunc stans in dem Entstehen zugleich Verschwinden bedeutet, was zugleich aber wieder Entstehen meint.

Insofern ist es dem Leerlauf des als reines Denken operierenden Automaten auch unangemessen, Übergänge als von einem Vorgänger- zu einem Nachfolgerzustand fortschreitende auszuweisen. Es gibt keinen konstatierbaren Fortschritt an Bestimmtheit, da das reine Denken, wie gesagt, keine fixierbaren Zustände besitzt, die das Feststellen(!) von (Fort)schritten erlauben würde. Das Gerichtetsein von... zu..., das selbst den Zirkel, die Schleife noch auszeichnet, ist im nunc stans des Entstehens, das zugleich Verschwinden ist, das zugleich Entstehen ist, ..., nicht zu verorten.

Hegel und auch Fichte weisen deshalb auch konsequenterweise darauf hin, dass es die W. d. L. bzw. die W.L. eigentlich nur mit einer Bestimmtheit, einem Begriff zu tun haben $^{17}$, so dass das ganze System nur in der einen laufenden Begriffsoperation sich macht und sich hat. Da es ihm an einer Laufzeitumgebung fehlt, kann es nicht verlassene Zustände als wieder erreichbare in der als Speichermedium genutzten Umgebung hinter sich festhalten. Das macht die Absolutheit des reinen Denkens aus, dass es vollständig umgebungsfrei $\mathrm{zu}$ operieren hat. Auch die extensionslose Zeit verweigert eine Ordnung des Vorher und Nachher, in der sich Bestimmtheiten fixieren lassen. Die Momente des Entstehens und Verschwindens, die das nur auf sich selbst operierende Denken durchläuft, sind auch im zeitlichen Sinne als Momente zu nehmen. Im Moment des Entstehens ist Verschwinden und umgekehrt. Die Redeweise, dass aus dem leerlaufenden Betrieb des Nicht-Bestimmens die Bestimmtheiten "Entstehen" und "Verschwinden" entwickelt werden, ist keine, die in der Exekution des Nicht-Bestimmens entsteht, weil darin ein identifizierender Rückgriff auf das als Ausgang bestimmte Nicht-Bestimmen erfolgen würde. Der Automat des reinen Denkens, so wie er hier konstruiert wurde, lässt sich als fortschreitender nur unangemessen beschreiben. Da er zustandslos ist, ist er streng genommen gar nichts. Genau als das realisiert ihn aber sein eigener Betrieb. Dem nunc stans, der Ewigkeit eignet es, dass darin keine zeitlichen Streckungen eingetragen werden können.

Bevor das dritte von der Theorie der Zustandsautomaten vorgegebene Thema, das eindeutige Ende, behandelt wird, welches sich in Anbetracht dessen dringlich stellt, dass im nunc stans des Nicht-Bestimmens sich kein Haltepunkt fixieren lässt, soll auf die Besonderheiten des Gedankenfortschritts in der W.L. eingegangen werden. Da sich die Behandlung des

${ }^{17}$ Vgl. Johann Gottlieb Fichte, Wissenschaftslehre 1812, S. 47: "Die W.-L. ist das schlechthin sich selbst machende apriorische Bild des Wissens in seiner absoluten Einheit und Gesetzmässigkeit: mithin auch Eins"; Georg Friedrich Wilhelm Hegel, Wissenschaft der Logik, S. 39: "Ausserdem aber muß die Notwendigkeit des Zusammenhangs und die immanente Entstehung der Unterschiede sich in der Abhandlung der Sache selbst vorfinden, denn sie fällt in die Fortbestimmung des Begriffs". 
Voraussetzungslosen, Umgebungsfreien in der W.L. von der der W. d. L unterscheidet, steht zu erwarten, dass ihr Vollzug, die Exekution der darin angelegten Programmatik sich anders gestaltet, als das Kontinuum, in dem Entstehen und Verschinden in eins fallen und somit mit ihrem Entstehen sogleich wieder verschwinden. Die Systementwicklung der W.L. gewinnt ihren Antrieb daraus, dass es nicht bei dem Widerspruch bleiben darf, das, zu dem es keine Umgebung gibt, sei als solches nur, weil es eine Umgebung gibt, aus der heraus seine Bestimmtheit ausgesagt wird. Dass das Gesetz des verbotenen Widerspruchs vorausgesetzt werden kann, ist Folge des qua Urteilsform als Jenseits aller gedanklichen Bestimmungen identifizierten In-Sichs. Der Widerspruch kann deshalb festgestellt werden, weil das, dem die Bestimmtheit, kein Außerhalb zu haben, zugesprochen wird, dasselbe ist wie das, dem die Bestimmtheit zugesprochen wird, ein Außerhalb zu benötigen, damit es als das bestimmt werden kann, das kein Außerhalb hat. Der Fortschritt in der Systementwicklung der W.L. wird dadurch erzielt, dass eine Konstellation gefunden wird, die den Widerspruch löst. Indem allem in der Sphäre des Bestimmens Bestimmten Sein abgesprochen werden muss, kann diese auch nicht außerhalb des absoluten In-Sich Seinsstatus beanspruchen. Das, was in der Sphäre des Bestimmens bestimmt ist, entspricht nichts in der Sphäre des Seins. Es ist nur im und vom Bild Gebildetes: ${ }^{18}$ Da aber das Programm des Begriffs "Bestimmen" fordert, dass das Bestimmen in Relation zu nicht aus dem Bestimmen stammenden Bestimmten steht, kann das Dilemma nur dann gelöst werden, wenn sich zeigen ließe, dass das nur in seiner eigenen Sphäre sich bestimmende Bestimmen dies nur ist, wenn es Bild des Seins ist. Kann die W-L dieses Zeigen als das Sichbestimmen des Bestimmens in seiner eigene Sphäre ausweisen, so wäre die Bestimmtheit des absoluten In-Sich gerechtfertigt, ohne das ein Übergriff in seine Sphäre nötig wäre. Zum Einen wäre die Hypothetizität in Kategorizität überführt, zum Anderen die absolute Trennung der Sphären aufrechterhalten.

Die Systementwicklung der W.L. geht konkret nun so vor, dass, ausgehend von dem widersprüchlichen Erscheinungsbegriff, dass die Erscheinung zum Einen von ihr Unabhängiges voraussetzt, das in ihr zur Erscheinung kommt, und zum Anderen keinen Durchgriff auf eine Sphäre außerhalb erlaubt, da nur die Erscheinung erscheinen kann, dieser Erscheinungsbegriff auf beiden widersprechenden Seiten Fortbestimmungen erfährt, die in einem konsistenten Erscheinungsbegriff enden sollen. Ob dieser erreichbar ist kann nur der Fortgang erweisen.

Den Fortgang der Systementwicklung machen also die Fortbestimmungen des Erscheinungsbzw. Bildbegriffs aus. Da die W.L. auch Begriffe als Bilder auffasst, kann gesagt werden, der Bildbegriff bilde sich darin selbst aus. Ähnlich dem Vorgehen der W. d. L. wird der Begriff dem Gesetz, dem Programm unterworfen, das er selbst ist. Der Begriff "Erscheinung" der die Bestimmtheit "In-Sich" außerhalb des von ihr Bestimmten ermöglicht, muss als solcher erscheinen können, soll nicht das Gesetz verletzt werden, dass alles Bestimmte, mithin Abgrenzbare und eines Äußeren Bedürftige aus der erscheinenden Erscheinung sein muss. Jede Lösung dieses begrifflichen Konstruktionsproblems muss wiederum als Fortbestimmung

${ }^{18} \mathrm{Zu}$ beachten ist, dass die Termini "Bilden" und "Bild" in der W.L weiter gefasst sind, als in dem hier thematischen Zusammenhang begrifflichen Bestimmens. Andererseits ermöglicht nur reines Denken die Exekution begrifflicher Programme an Begriffen, und das, was man in Ausführung eines begrifflichen Programms tut, wieder unter Begriffe zu bringen. Im "Fortschreiten" der hier interessierenden Systeme ist nur das von Interesse. 
des Erscheinungsbegriffs also als Erscheinung der Erscheinung erscheinen. Somit werden Aussagen über die Erscheinung als Erscheinungsformen der Erscheinung selbst aussagbar und müssen konsistent sein mit den vorhergehenden Erscheinungsformen. Da man es in der gesamten W.L. mit Begriffen zu tun hat - der Ausgangspunkt wird ja faktisch beim Begriff des In-Sich genommen und nicht beim In-Sich selbst - darf man auch formulieren, dass die W.L. sicherstellen will, dass die gedachten Bestimmtheiten (Objekt) auch bestimmend sind für das Denken, das diese Bestimmtheiten denkt (Subjekt). Angestrebt wird also wie in der W. d. L. ein Konstrukt, dessen Bestimmungen nicht von außen an es herangetragen werden, sondern als im Betrieb des Konstrukts entstehend auszuzeichnen sind.

Im Gegensatz zur W. d. L., deren Betrieb aufgrund des radikalen Verzichts auf vorgegebene Bestände im nicht identifizierenden Leerlauf des nunc stans aufgeht, lässt die W.L. den Begriff des Absoluten, der die Konstruktionsaufgabe eines von allem Vorgegebenen Abgelösten inhaltlich ja erfüllt, in Form eines vorgefundenen Faktum bestehen. Wenn auch in der W.L. die Fortbestimmung des Erscheinungsbegriffs nicht auf ein von außen vorgegebenes Programm zurückgreift, das als Leitfaden dient, so hat sie im fixierten Begriff des Absoluten, der etwas in der Sphäre der Erscheinung ist, doch ein Maß, mit dem alle sich ergebenden Bestimmungen der Erscheinung sich widerspruchslos zusammenbringen lassen müssen. Die Aufhebung der Widersprüche geschieht dadurch, dass sich die widersprechenden Bestimmtheiten nicht mehr auf die eine Erscheinung beziehen, sondern nur auf unterschiedlichen Stufen, in denen sich die Erscheinung selbst erscheint ${ }^{19}$ und die nebeneinander Bestand haben. Die nebeneinander bestehenden Teilbestimmtheiten bedürfen dann eines neu bestimmten Einheitsbegriffs der Erscheinung, in dem sie zusammen bestehen können.

Mit dem im Ausgang fixierten Begriff des Absoluten übernimmt die W.L. auch das Operieren in Prädikationen, in dem sich das Denken als fremdreferentiell orientiertes immer schon vorgegeben ist. ${ }^{20}$ Der Fortgang der W.L. über zu lösende Widersprüche bedarf der Prädikation geradezu, da sich ausschließende Bestimmtheiten nur dann zum Verschwinden gebracht werden müssen, wenn sie auf ein identisches Bestimmtes zutreffen sollen. Zwar kann die Konsistenzanforderung nichts zur Genese der Bestimmtheiten der Erscheinung beitragen, aber sie gibt ein Kriterium ab, wann die Fortbestimmung der Erscheinung ihren Endzustand erreicht hat. Das geschieht dann, wenn sich die Erscheinung konsistent als Erscheinung des Absoluten bestimmen lässt, obwohl das, was erscheint, nur die Erscheinung selbst ist. Wenn jedes Bild als Fortbestimmung des Bildes des Absoluten erwiesen werden kann und das in der W.L. vollzogene Denken selbst als Bilden von Bildern auszuweisen ist, dann ist die Selbstabbildung des einen und einzigen Bildes, nämlich das des Absoluten, vollendet. Der Automat, der die W.L. ist, kann anhalten, weil er seine eigene Bestimmtheit als Output seines Betriebes ausgeworfen hat. Seine Bestimmtheit ist dem Automaten folglich nicht von außen vorgegeben, sondern Resultat seines eigenen Operierens. Das Faktum, dass

\footnotetext{
${ }^{19}$ Sehr deutlich ist die Disjunktion der Ebenen, auf die die widersprechenden Bestimmtheiten verteilt werden am Schema-Konzept der W.L. abzulesen. Ein Schema repräsentiert die Form in der das Erscheinen der Erscheinung rein begrifflich gefasst wird: seiende Erscheinung, Erscheinung der Erscheinung, Erscheinung der Erscheinung als Erscheinung der Erscheinung.

${ }^{20}$ Fichte formuliert Dreh- und Angelpunkte der Entfaltung der W.L. häufig als Sätze. Vgl. z. B. Johann Gottlieb Fichte, Wissenschaftslehre 1812, S. 58: "Ist der absolut bejahende Satz der W.L."
} 
die Erscheinung da sein muss, damit sie sich als Erscheinung des Absoluten, welches sie nicht selbst ist, bestimmen kann, bleibt allerdings dem Vollzug der W.L. vorgegeben. Die Bestimmtheit der Erscheinung, die eine unmittelbare Erscheinung des Absoluten zu sein, besagt zwar, dass jede Bestimmtheit, die auftreten kann und somit alles, dem Dasein zugesprochen werden kann, auf die eine Erscheinung muss rückführbar sein, aber sie selbst kann nur erscheinen, weil Erscheinung faktisch immer schon im Vollzug ist. Letztendlich bleibt also in der Entwicklung der W.L. der für den Anfang konstatierte Restbestand ${ }^{21}$ an Vorgegebenheit auch am Ende in der untilgbaren Faktizität bestehen. Bei aller Betonung von Selbstreferenz liegt der W.L. letztendlich das sich im Verhältnis von Bild und Sein aussprechende Konzept von Fremdreferenz zugrunde. Davon tangiert wird selbst noch die Fassung der Selbstreferenz, die als Sichabbildung eines bereits seienden Bildes verstanden wird.

Während in der W.L. über den als Norm für die Unabhängigkeit von Vorgegebenem fungierenden Begriff des Absoluten ein Kriterium festgelegt ist, bei dem der Lauf des Automaten ein Ende finden kann, kann in der W. d. L. auf ein solches Kriterium nicht zurückgegriffen werden.

\section{Das Ende}

Der Betriebsmodus der W. d. L., indem er jenseits der sich in der Bestimmung eines bestimmt Vorgegebenen abschließenden Prädikation operiert, kann nämlich überhaupt keine identifizierbare und somit sich identisch durchhaltende Bestimmtheit zur Verfügung stellen. Dem modus operandi der W. d. L., der sich dem radikalen Verzicht auf irgendeine Vorgegebenheit verdankt, ist zu eigen, dass Bestimmtheiten nicht zu identifizieren sind, da sie und das, was sie nicht sind, nicht auseinandergehalten werden können. Wie sich in den ersten Drehungen ${ }^{22}$ des Automaten gezeigt hat, gehen die Bestimmtheiten jeweils in ihrem Gegenbegriff auf: Bestimmtheit ist einzig Unbestimmtheit, Entstehen ist einzig Verschwinden. Das Ineinanderaufgehen bedarf zwar genauso des momentanen Unterschieds, weil ansonsten das Gegen des Gegenbegriffs gar nicht möglich wäre. Dieses Moment auftretender Bestimmtheit ist aber so lange zum Verschwinden verurteilt, wie die gegeneinander Gesonderten ineinander aufgehen.

Da bereits die soeben getätigte Beschreibung des Beginns der W. d. L. keine vom automatischen Vollzug angefertigte ist, müssen auch die folgenden Überlegungen zu einem möglichen Ende in die inadäquate Theorie gerechnet werden, die den Betrieb des Automaten als etwas Vorgegebenes, Identifizierbares nimmt. Der Verbrauch immer neuer nur momentan auftauchender Bestimmtheiten, die ihren Bestimmtheitscharakter dadurch verlieren, dass das, was sie bestimmen, ihr Gegenteil ist, wäre nur dann zu beenden, wenn die für das

\footnotetext{
${ }^{21} \mathrm{Vgl}$. oben, S. 375.

${ }^{22}$ Die Ausdrucksweise "erste Drehung" ist sowohl von der Verwendung des Zahlausdrucks als auch von dem als Merkmal von Bewegung genutzten Ausdruck "Drehung" her keine im Vollzug der cogitatio entstehende Bestimmtheit. Da aber die Erwägungen zum möglichen Haltepunkt des Betriebs der W. d. L. ihr auch nicht zuzurechnen sind, muss dieser Ausdruck genügen, um auf das Gemeinte zu verweisen.
} 
Bestimmtsein erforderliche Sonderung, nicht durch eine neu auftretende Bestimmtheit ausgeführt wird, sondern die eine ungesonderte Einheit, in der die eine Bestimmtheit zugleich Bestimmtheit ihrer Negation und somit von absolut unspezifischer Allgemeinheit ist, eodem actu die eine Besonderung ist, die der einen Einheit Bestimmtheit verleiht. Darin wäre die eine Einheit ein durch sich selbst bestimmtes, wäre Einzelnes, das nicht auf den ihm vorgegebenen Raum als principium individuationis angewiesen ist. In ihrer Vereinzelung wäre die einzig durch sich bestimmte Einheit auch Einziges.

Aufgrund des "Ausgangs" mittels der Befreiung von jeder vorgegebenen Bestimmtheit gelänge die Konstruktion einer Selbstbeziehung, die nicht damit zu kämpfen hätte, von einem bereits Vorliegenden ausgehen zu müssen, das seine Bestimmtheit dann eben nicht aus der Selbstbeziehung erhält, sondern sie bereits besitzt, um dann von außen die weitere Bestimmtheit zugesprochen zu bekommen, dass sie sie aus sich hätte. ${ }^{23}$ Voraussetzung für eine umgebungsfreie nur sich aus sich machende Selbstbeziehung, ist das Operieren der cogitatio jenseits aller Positivität, in der Leere der absoluten Negativität, die die Positionierung der Negation (des Nichts) gegenüber der Position (dem Sein) selbst noch für nichtig erklärt.

Was die Systeme der W.L. und der W. d. L. bei aller Verschiedenheit auszeichnet, ist ihr Vertrauen in den reinen, nicht auf empirisch Vorgegebenes bezogenen Begriff. Beide trauen ihm zu, aus sich zu entfalten, was er ist. Was Denken ist, kann sich nur im Vollzug dieses Denkens selbst erweisen. Autonom operieren kann ein solches Denken nur, wenn es Begriffe findet, die nur auf sich applikabel sind. Während die W.L. diesen Begriff in "Bild" findet, ist dies für die W. d. L. die Leere des Unbestimmten, die absolut gesetzte Negation.

${ }^{23}$ M. E. kommt es aufgrund des Festhaltens an den im Zusprechen des Prädikats sich abschließenden und fixierenden Prädikationen in der W.L. dazu, dass Selbstbeziehung zwar z. B. mittels des Prädikats "SichErscheinung" gemeint ist, aber dann im Betrieb nicht konstruiert werden kann,. Es liegt ein qua logisches Subjekt identifiziertes Einzelnes vor, dessen agierender Selbstbezug, aber nicht die beschreibende Prädikation selbst ist. Selbst wenn das Urteil "Die Erscheinung erscheint sich." als das qualifiziert wird, worin die Erscheinung sich erscheint, bricht die Differenz von fixiert Vorliegendem und hinzutretendem Prädikat wieder auf: Die Erscheinung ist sich-erscheinend als Erscheinung, die sich-erscheinend ist. Insofern bleiben Programmausführung und -bebeschreibung auf getrennten Ebenen und werden nur in der satzförmigen Beschreibung, die Programmbeschreibung sei Ouput der Exekution des Programms, extern zusammengebracht. 